PROCEEDINGS OF THE AMERICAN MATHEMATICAL SOCIETY

Volume 124, Number 10, October 1996

\title{
INFINITE CYCLIC VERBAL SUBGROUPS OF RELATIVELY FREE GROUPS
}

\author{
A. STOROZHEV
}

(Communicated by Ronald M. Solomon)

\begin{abstract}
We prove that there exist a relatively free group $H$ and a word $w(x, y)$ in two variables such that the verbal subgroup of $H$ defined by $w(x, y)$ is an infinite cyclic group whereas $w(x, y)$ has only one nontrivial value in $H$.
\end{abstract}

In [6] (see also [2], Problem 2.45a), the following question of P. Hall is stated. Is it true that if there is only finitely many different values of a word $v$ in a group $H$, then the verbal subgroup $v(H)$ is finite? S. Ivanov [1] answered this question in the negative; he constructed a group $H$ and a word $v(x, y)$ such that $v(H)$ is infinite cyclic but $v(x, y)$ has only one non-trivial value in $H$. In [1], he also asked about what may happen if extra conditions are imposed on $H$. In particular, he raised the problem as to whether the fact that there are finitely many values of a word $v$ in a relatively free group $H$ implies the finiteness of the verbal subgroup $v(H)$. He repeated this question later in [3]. It is interesting to note that the construction described in [5] shows that there exists a word $u(x, y)$ and a relatively free group $K$ such that $u(K)$ is infinite cyclic and all the elements of $u(K)$ are values of the word $u(x, y)$ in $K$. In this paper we give the negative answer to the question raised by S. Ivanov.

We put

$$
\begin{gathered}
u=u(x, y)=\left(x^{d} y^{d}\right)^{d} x^{d}, \\
v=v(x, y)=\left[u^{d}, x^{d}\right], \\
w(x, y)=u^{\varepsilon_{1}} v^{n+1} u^{\varepsilon_{2}} v^{n+2} \cdots u^{\varepsilon_{h}} v^{n+h},
\end{gathered}
$$

where $h \equiv 0(\bmod 10), \varepsilon_{10 k+1}=\varepsilon_{10 k+2}=\varepsilon_{10 k+3}=\varepsilon_{10 k+5}=\varepsilon_{10 k+6}=1, \varepsilon_{10 k+4}=$ $\varepsilon_{10 k+7}=\varepsilon_{10 k+8}=\varepsilon_{10 k+9}=\varepsilon_{10 k+10}=-1, k=0,1, \ldots, h / 10-1$ and $d, n, h$ are sufficiently large natural numbers chosen with respect to the restrictions that are introduced in Chapter 7 of [4].

Let $G$ be an arbitrary group; then $w(G)$ denotes the verbal subgroup of $G$ defined by the word $w(x, y)$.

Theorem. There exists a relatively free group $H$ such that the verbal subgroup $w(H)$ is an infinite cyclic group whereas $w(x, y)$ has only one nontrivial value in $H$.

Received by the editors March 6, 1995 .

1991 Mathematics Subject Classification. Primary 20E10, 20 F06.

(C)1996 American Mathematical Society 
Proof. Let $F_{2}$ be the absolutely free group of rank two. The word $w(x, y)$ coincides with one of the words $w_{m}(x, y), m=1,2, \ldots$, introduced in [5], namely $w_{1}(x, y)$. Therefore, when studying the group $w\left(F_{2}\right)$ and groups associated with it, we can use lemmas proved in [5]. In particular, by Lemma 17 of [5], all the nontrivial values of $w(x, y)$ in $\bar{G}=F_{2} /\left[w\left(F_{2}\right), F_{2}\right]$ form a basis of the free abelian group $w(\bar{G})$. Now let $V$ be the subgroup of $\bar{G}$ generated by all the elements of the form $w(X, Y)$ and $w\left(X_{1}, Y_{1}\right)\left(w\left(X_{2}, Y_{2}\right)\right)^{-1}$ for all pairs $(X, Y),\left(X_{1}, Y_{1}\right)$ and $\left(X_{2}, Y_{2}\right)$ of words such that the images of the words $X$ and $Y$ do not form a basis of the free abelian group $F_{2} /\left[F_{2}, F_{2}\right]$ and the images of the words in each of the pairs $\left(X_{1}, Y_{1}\right)$ and $\left(X_{2}, Y_{2}\right)$ form a basis of $F_{2} /\left[F_{2}, F_{2}\right]$. By Lemma 10 of [5] and Lemma 15 of [5], the fact that $w\left(S_{1}, T_{1}\right)=w\left(S_{2}, T_{2}\right)$ in $\bar{G}$ yields that $S_{1}$ is conjugate to $S_{2}$ and $T_{1}$ is conjugate to $T_{2}$ in the group $F_{2} / w\left(F_{2}\right)$. Hence $S_{1}=S_{2}$ and $T_{1}=T_{2}$ in $F_{2} /\left[F_{2}, F_{2}\right]$. Therefore, $V$ is a verbal subgroup of $\bar{G}$ and the group $H=\bar{G} / V$ is a relatively free group such that the image of $w\left(F_{2}\right)$ in $H$ is an infinite cyclic group. It is also clear that there is at most one nontrivial value of the word $w(x, y)$ in $H$. Thus the Theorem is proved.

\section{REFERENCES}

[1] S.V. Ivanov, P. Hall's conjecture on the finiteness of verbal subgroups, Izv. Vyssh. Ucheb. Zaved. 325 (1989), 60-70. MR 90j:20061

[2] Kourovka Notebook, Unsolved problems of the group theory, Tenth Edition, Novosibirsk, 1986.

[3] Kourovka Notebook, Unsolved problems of the group theory, Eleventh Edition, Novosibirsk, 1991.

[4] A.Yu. Ol'shanskii, Geometry of defining relations in groups, Mathematics and Its Applications (Soviet Series), vol. 70, Kluwer Academic Publishers, Dordrecht, 1991. MR 93g:20071

[5] A. Storozhev, On abelian subgroups of relatively free groups, Comm. Algebra 22 (1994), 2677-2701. MR 95d:20066

[6] R.F. Turner-Smith, Marginal subgroup properties for outer commutator words, Proc. London. Math. Soc. 14 (1964), 321-341. MR 29:2289

Australian Mathematics Trust, University of Canberra, PO Box 1, Belconnen, ACT 2616, Australia

E-mail address: ans@amt.canberra.edu.au 